\title{
Strategies for integrating Sleep Medicine in Dental Practice and Postgraduate Training
}

\author{
${ }^{1}$ Abhijeet Kadu, ${ }^{2}$ Balakrishnan Jayan, ${ }^{3}$ Reena R Kumar, ${ }^{4}$ Oommen Nainan, ${ }^{5}$ Probod K Chattopadhyay
}

\begin{abstract}
Dentistry's entry into the management of upper airway sleep disorders has led to the development of a new specialty, i.e., dental sleep medicine. This specialty involves oral appliance therapy (OAT) for obstructive sleep apnea (OSA) and snoring, maxillomandibular advancement surgeries to increase upper airway volume, assessment of craniofacial risk factors, cephalometric studies for craniofacial and airway evaluation, and prevention of sleep-disordered breathing (SDB) in children. Dentists trained in dental sleep medicine, orthodontists, and maxillofacial surgeons are recognized members of interdisciplinary team to manage upper airway sleep disorders. So it is of paramount importance for dental students and orthodontists to familiarize with the basic knowledge of upper airway sleep disorders and its management in order to contribute effectively in managing those affected patients. There is need to integrate dental sleep medicine in postgraduate orthodontic curriculum. The current status of this new and challenging discipline in India, the Armed Forces Medical College and Army Dental Centre (Research and Referral) experience, and strategies to build capacity are discussed in this article.
\end{abstract}

Keywords: Dental practice, Postgraduate training, Sleep medicine.

How to cite this article: Kadu A, Jayan B, Kumar RR, Nainan O, Chattopadhyay PK. Strategies for integrating Sleep Medicine in Dental Practice and Postgraduate Training. Indian J Sleep Med 2017;12(3):33-38.

Source of support: Nil

Conflict of interest: None

\section{INTRODUCTION}

About $7.5 \%$ of urban Indian population is estimated to be chronic sufferers from upper airway sleep disorders

${ }^{1}$ Graded Specialist, ${ }^{2}$ Consultant, ${ }^{3}$ Dean and Head, ${ }^{4}$ Classified Specialist, ${ }^{5} \mathrm{Head}$

${ }^{1,2}$ Department of Orthodontics and Dentofacial Orthopedics Army Dental Centre (Research and Referral), New Delhi, India

${ }^{3}$ Department of Orthodontics and Dentofacial Orthopedics, Divya Jyoti College of Dental Sciences \& Research, Modinagar, Uttar Pradesh, India

${ }^{4}$ Department of Orthodontics, Office of Director General Medical Services (Navy), New Delhi, India

${ }^{5}$ Department of Oral and Maxillofacial Surgery, Army Dental Centre (Research and Referral), New Delhi, India

Corresponding Author: Abhijeet Kadu, Graded Specialist Department of Orthodontics and Dentofacial Orthopedics, Army Dental Centre (Research and Referral), New Delhi, India, Phone: +919971530711, e-mail: drabhijeetkadu@gmail.com and many of them have impaired health and daily functional issues as a result of these disorders. ${ }^{1}$ The societal economic impact is humongous and unfortunately 82 to $98 \%$ are undiagnosed.

The evolution of hominids leading to modern man has resulted in jaws playing a vital role in respiration and speech. $^{2}$ The profession of orthodontics and dentofacial orthopedics which primarily deals with teeth and jaws aims at achieving structural balance, functional harmony, and optimization of dentofacial esthetics. Enhancing upper airway dimensions and overcoming its collapsibility in sleep by correct spatial orientation of the jaws is integral to functional harmony which is often ignored and not adequately addressed during postgraduate training and subsequent practice. A change in the mindset and revised approach to orthodontic practice is the need of the hour so that the management of upper airway sleep disorders can be an integral part of our day-to-day practice and we all become rightful stakeholders. Scope and strategies toward capacity building would be analyzed and futuristic and pragmatic solutions would be put forward vide this communication.

\section{DISCUSSION}

\section{Scope and Brief Overview}

The last 50 years have seen remarkable advances in the field of circadian biology and neurophysiology of sleep. The genes that regulate these biologic rhythms have been isolated and there are interactions between sleep and all other body systems, namely respiratory, cardiovascular, endocrine, and neurologic. ${ }^{3}$ These scientific advances have emanated from diverse clinical disciplines including internal medicine, pulmonology, neurology, otorhinolaryngology, psychiatry, and nursing. The range of specialties reflects interdisciplinary nature of sleep and its disorders, and many critical contributions have come from various disciplines of dental sciences namely orthodontics, maxillofacial surgery, and prosthetic dentistry. Currently, approximately 100 distinct clinical sleep disorders have been recognized; of these, certain disorders like OSA, sleep bruxism, and chronic orofacial pain have a direct bearing on dental practice in general and orthodontic practice in particular. ${ }^{4}$ This makes a working understanding of somnology and sleep medicine a useful and necessary addition to the knowledge base of orthodontists. 
Sleep disorders decrease the quality of life and cause mood alterations, memory problems, and workplace performance deficits. It grossly affects daytime vigilance, resulting in increased risk of transport or work-related accidents. In the long run, OSA is known to be a serious and modifiable risk factor for cardiovascular disease including heart failure and stroke. ${ }^{5,6}$ The intrusion of snoring and tooth grinding sounds is also a major cause of sleep disruption for the patient's bed partner and can be a source of martial conflict. Orofacial pain may be associated with delayed sleep onset and fragmented sleep, resulting in insomnia that may predispose patients to mood alterations and depression. ${ }^{7}$

Sleep medicine is often an overlooked part of public health. In many countries, access to sleep medicine constitutes a major public health challenge. In countries where therapy is available, treating sleep disturbances either as primary disorders or as comorbidities with other medical, psychiatric, or dental conditions is a significant opportunity to prevent medical and psychiatric morbidity. It may also minimize the substantial financial burden related to the direct and indirect consequences of disturbed sleep. ${ }^{8}$

Dentists in general and orthodontists in particular play an important role in sleep medicine by examining patients during their annual or biannual dental checkup for the risk of SDB. Patients reporting snoring, sleepiness, and morning headaches in the presence of obesity, large tonsils, and/ or dental malformation (e.g., retrognathia, deep palate, large tongue) need to be guided by dental practitioners to see their otorhinolaryngologist, pulmonologist, or physician as well as a sleep medicine expert. To manage the sound and tooth damage or pain generated by bruxism, oral appliances can be used, but the orthodontist needs to understand when such an appliance is indicated and the risks associated with its use. In cases where surgery is indicated, maxillofacial surgeons or otorhinolaryngologists collaborate closely with orthodontists to provide treatment.

When patients complain of morning headaches and temporomandibular disorders (TMDs), the exclusion of breathing disorders is a critical decision that is usually made in collaboration with the sleep medicine specialist, pulmonologist, neurologist, psychiatrist, and internal medicine physician. Orthodontists should refer patients who experience sleep bruxism in combination with a TMD for polysomnographic evaluation when they also complain of significant insomnia or poor sleep, even if they do not meet the traditional risk factors for sleep apnea. ${ }^{9}$ An increasing body of data suggests that both sleep bruxism and TMDs which often occur in females are associated with increased risk for SDB.

Orthodontists caring for patients with chronic orofacial pain conditions (such as TMDs) also need to under- stand basic sleep hygiene principles and to know when to refer patients with chronic or intractable insomnia for behavioral sleep medicine evaluation. Behavioral treatments for chronic insomnia are considered first-line interventions over pharmacologic treatment options. A subset of chronic orofacial pain patients present with a complex psychologic overlay that contributes to their ongoing pain and disability, a combination that can be managed by sleep psychologists working in conjunction with the interdisciplinary team.

There is mounting evidence with respect to craniofacial anomalies like mandibular hypoplasia, maxillary deficiency, long face syndrome, deep and narrow palate, and inferiorly and posteriorly placed hyoid bone as risk factors for developing SDB, particularly OSA. ${ }^{4,10-12}$ Orthodontists are trained to handle these anomalies but often they factor only the esthetic component. If these skeletal malocclusions are appropriately addressed by preventive, interceptive, and corrective orthodontics, the spin-offs in the form of neutralizing craniofacial risk factors for SDB would be crucial and paramount. The negative impact of extractions in orthodontics on upper airway will warrant a relook into our traditional treatment options as extractions can reduce oral volume and cramp up tongue space. ${ }^{13}$

Oral appliances particularly mandibular advancement appliances have been endorsed by the American Academy of Sleep Medicine as the treatment option for nonapneic snoring, mild to moderate OSA cases, and severe cases of OSA not amenable to continuous positive airway pressure (CPAP) therapy and surgery. ${ }^{14,15}$ Many studies have shown that patients prefer OAT over CPAP. ${ }^{16,17}$ The clinical and laboratory procedures for mandibular advancement device (MAD) are same as functional appliances like activator or a twin block. Thus, for an orthodontist, management of snoring and OSA with oral appliances is going to a very easy transition. Maxillomandibular advancement surgeries are considered an almost 100\% cure after tracheotomy. ${ }^{18}$ The maxillomandibular surgical procedure is executed vide orthognathic surgery or distraction osteogenesis which requires a close collaboration of orthodontists and maxillofacial surgeon.

It is high time that the dental fraternity realize that dental sleep medicine is a rapidly evolving field of preventive medicine. However, there remains a shortage of welltrained dental sleep medicine specialists. Those learning more about this field will discover an exciting interdisciplinary arena that is rife with opportunities to develop new dental interventions to treat complex clinical situations and improve the health and well-being of the estimated $20 \%$ of the population suffering from sleep disorders.

Ivanhoe and Attanasio ${ }^{19}$ nearly a decade back stated that upper airway sleep disorders are potentially life threatening, the ability to treat patients may be critical. 
Dentists in general and orthodontists in particular are recognized as an integral part of the treatment team of these patients. Even though numerous studies and publications are available, most dental schools do not include the treatment of upper airway sleep disorders as a part of the curriculum, so most of the dental professionals are not able to manage these disorders.

A Google search shows that 24 articles on OSA have been published in leading orthodontic journals alone, namely American Journal of Orthodontics and Dentofacial Orthopedics (11), European Journal of Orthodontics (7), International Journal of Orthodontics (2), Journal of Clinical Orthodontics (2), in the last 5 years. The impact on orthodontic practice in the West has been profound. Dental sleep societies have emerged in the United States and Europe. Certification and fellowship programs have been introduced. Webinars of dental sleep medicine initiated in Sleep Group Solutions is great initiation in distance education which can be leveraged by orthodontists around the word to enhance their skills and knowledge. ${ }^{20}$

Leading universities, such as Stanford, Bristol, and Sydney have incorporated management of OSA in the university curriculum. Research on the impact of orthodontic treatment procedures like rapid maxillary expansion (RME), maxillomandibular surgeries, extraction orthodontics, functional jaw orthopedics on upper airway capacity are being carried out in these universities. In India the practice of dental sleep medicine started nearly a decade earlier in the Armed Forces wherein a case of severe mandibular hypoplasia secondary to bilateral temporomandibular joint ankylosis with severe OSA was successfully addressed by intraoral mandibular distraction osteogenesis in 2002. ${ }^{21}$ Fixed and titrable MAD for the management of OSA and nonapneic snoring was introduced by the Armed Forces group in 2004. The group has published several papers related to dental sleep since then. ${ }^{12,22-27}$ There are a number of articles in leading newspapers, health magazines on OSA and other sleep disorders which has, to a great extent, sensitized our masses and health care professionals. In addition to the above cited studies, there are only three other Indian studies on dental sleep medicine reported as per internet search indicating a wide gap.

\section{Curriculum Revision, Infrastructure Needs, and Interdisciplinary Approach}

With nearly 300 dental schools in India, the scope for research and practice in the field of dental sleep medicine, upper airway studies, and imaging is tremendous. There is a case in point to introduce workshops on dental sleep medicine during state, national, and postgraduate conventions for the next 5 years to build capacity. Dental schools can harness the experience and expertise of dental members of Indian Sleep Disorders Association to run basic and advance programs in dental sleep medicine. This group of authors has already organized dental sleep medicine workshops at annual national conferences of Indian Sleep Disorders Association (SLEEPCON) for last 3 years at different places in India. The participants in the workshop include dentists of all specialties and response is encouraging every year. On an average around 50 participants across the country have attended the workshops every year which aimed at understanding the basics of sleep, dental sleep medicine, details about OAT, hands-on demonstration of bite registration and fabrication of oral appliance, various diagnostic modalities available with dentists, and prevention of SDB in children (Fig. 1 and Table 1). In the workshop, intense and interactive training was given for 8 to 9 hours depending on duration and scheduling of 1 or 2 days. At the end of the workshop, feedback of the participants was sought, which unanimously accepted the importance of knowledge about sleep medicine in contemporary dental practice and necessity for increasing the frequency of such workshops to spread awareness among dentists. Most of the participants found the hands-on and audiovisual demonstration of bite registration and oral appliance fabrication as the most valuable aspect of the workshop. Many participants expressed their concern about lack of preliminary knowledge about sleep dentistry in our undergraduate as well as postgraduate curricula. Participants believed that the workshop will improve their perspective toward dental practice and their base knowledge has increased which is evident from the take-off test scores (11.5) at the beginning of the course and end of course (17) undertaken by all the participants on 25 objectively designed questions based on the course contents. The group made an endeavor to train manpower in the form of dental technicians so that oral appliances can be easily made and capacity should be built in the dental labs also to take on the ever-increasing load of oral appliances for management of SDB.

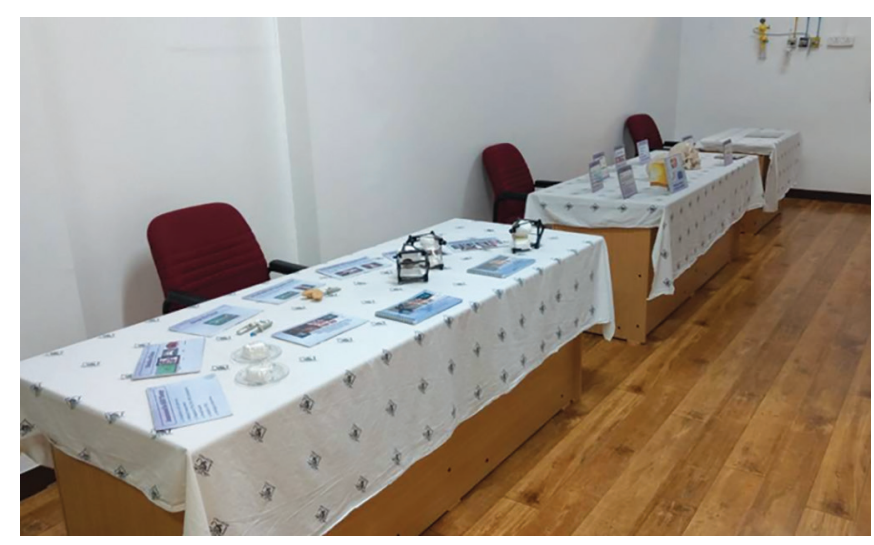

Fig. 1: Setup for demonstration of hands-on bite registration during SLEEPCON 2017 
Table 1: Contents of the dental sleep medicine workshop

\begin{tabular}{ll}
\hline Timing & Title \\
\hline $30 \mathrm{~min}$ & Take-off test \\
$30 \mathrm{~min}$ & Basics of sleep \\
$60 \mathrm{~min}$ & Dental sleep medicine: an overview \\
$40 \mathrm{~min}$ & Diagnostic considerations in upper airway sleep disorders \\
$60 \mathrm{~min}$ & Oral appliance therapy for snoring and OSA \\
$30 \mathrm{~min}$ & OASYS appliance therapy \\
$30 \mathrm{~min}$ & Summing up and Q\&A session \\
$30 \mathrm{~min}$ & Demonstration of recording of bite with George \\
& Bite Gauge \\
$90 \mathrm{~min}$ & Integrated table clinics. Display of various OAs for \\
& management of snoring and OSA. End-of-course test
\end{tabular}

The orthodontic postgraduate should have a 1-week attachment in the Department of Pulmonology and Sleep Medicine to have a working knowledge of spirometery, polysomnography, and CPAP titration. An attachment of 1 week in the Department of Otorhinolaryngology should help orthodontic postgraduates to be able to correctly examine tonsils, adenoids, septal deviations, turbinate hypertrophy and for familiarity of nasopharyngoscopy. Postgraduate departments should have access to polysomnography and rhinometery as these are basic and integral to practice of dental sleep medicine and evaluation of upper airway. Didactic lectures by sleep medicine specialists and otolaryngologists for postgraduate students in orthodontics is the need of the hour.

Patients with SDB are not likely to consult an orthodontist but most always a pulmonologist and otorhinolaryngologist. There is a case in hand to sensitize the medical fraternity of the role of dentistry in sleep medicine. As a protocol, all the cases of OSA may be referred by the pulmonologist for mandatory ear, nose, and throat (ENT) evaluation and craniofacial evaluation by an orthodontist. Upper airway imaging vide cephalometry, magnetic resonance imaging or computerized tomography scan must be made integral and mandatory. Landmark research studies at Stanford University have shown the benefits of combined RME and adenotonsillectomy in the management of sleep disorders in children. ${ }^{28,29}$ So there is a case in hand for all cases of adenotonsillar hypertrophy to be subjected to sleep study and orthodontic evaluation for holistic management. Practitioners, postgraduate teachers, and students should and always include airway grading (Mallampati), presence of snoring, Epworth sleepiness scale, airway analysis on cephalogram in all the cases. This will bring about change in attitude and modification in behavior and holistic appreciation of the orthodontic case. Two books on dental sleep medicine may be included in the syllabus and recommended for postgraduate students, namely Dental Management of Sleep Disorders authored by Attanasio and Bailey ${ }^{4}$ (Wiley
Blackwell 2010) and Sleep Medicine for Dentists: A Practical Overview by Lavigne et $\mathrm{al}^{3}$ (Quintessence Publishing Co, Inc). The way forward is to introduce integrated teaching and practice in dental schools and revision of postgraduate curriculum. Postgraduate students should be encouraged to take up upper airway-related studies as dissertations and to exhibit cases related to the same during the Master of Dental Surgery practical examinations. The same may be shown in lieu of functional case.

\section{Armed Forces Medical College and Army Dental Centre (R\&R) Experience}

In the Orthodontic Department of Armed Forces Medical College we have been successful in introducing dental sleep medicine. The department has leveraged the expertise of the Department of Pulmonology and Otorhinolaryngology for the same. Lectures on dental sleep medicine have been introduced for postgraduate students pursuing MD (Pulmonology), which has sensitized the students on craniofacial risk factors, OAT, RME, and maxillomandibular advancement surgeries. The Department of Pulmonology follows a protocol of mandatory screening of all cases for ENT and orthodontic evaluation. All the cases reporting for orthodontic treatments are screened for upper airway sleep disorders. All the cases of maxillary and mandibular advancement surgery undergo polysomnography at baseline and after treatment. We have introduced the following inclusion criteria for OAT for OSA, which has improved our success rate.

Mild (AHI: 5 to 15), moderate (AHI: 15 to 30), and severe (AHI: 30 and above) not amenable to CPAP therapy and $\mathrm{BMI}<30$

Any of the two positive cephalometric findings and model analysis:

- $\mathrm{ANB}>6^{\circ}$ with decreased SNB

- Decreased SNA and condylion to Point A distance

- Increased mandibular plane to hyoid bone (hyoid distance) (>15 mm)

- Deep palate

- Narrow maxillary arch with cross-bite.

We prescribe Karwetzky activator (fixed MAD) (Fig. 2), third-generation adjustable MAD (medical dental snoring appliance) (Fig. 3), mandibular advancement splint with tongue lifters and nasal dilators (Fig. 4). The later appliance is prescribed when there is enlarged tongue with crenations on the lateral border and small nares. We have introduced combined OAT with indirect hyoid suspension and prophylactic glossotomy in mandibular setback cases of more than $7 \mathrm{~mm}$. A total of 56 cases of OSA in the last 4 years have been successfully managed with OAT. The group is following American Association 

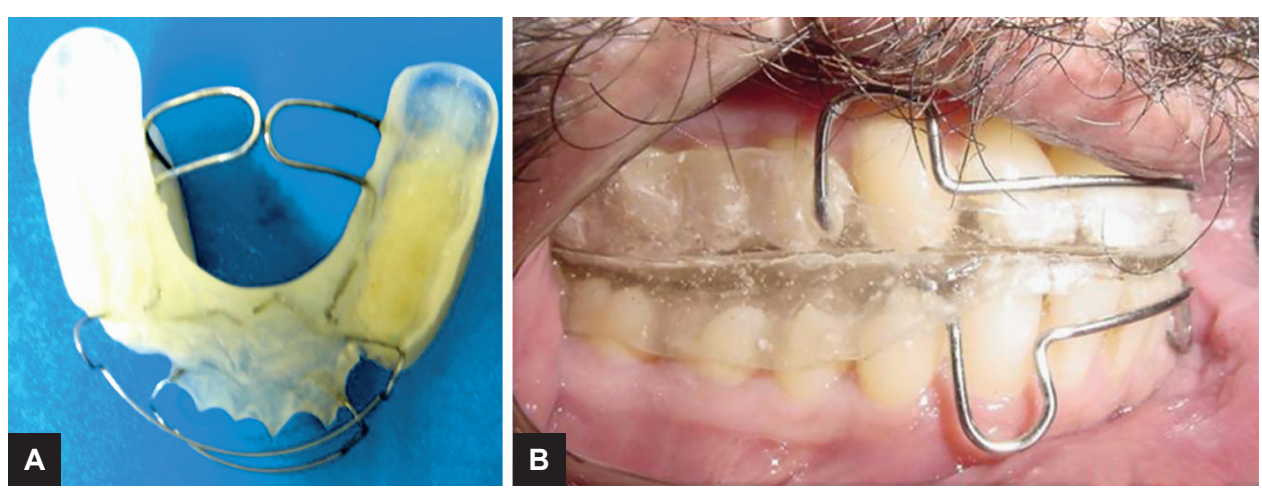

Figs 2A and B: Karwetzky activator (fixed titratable MAD)
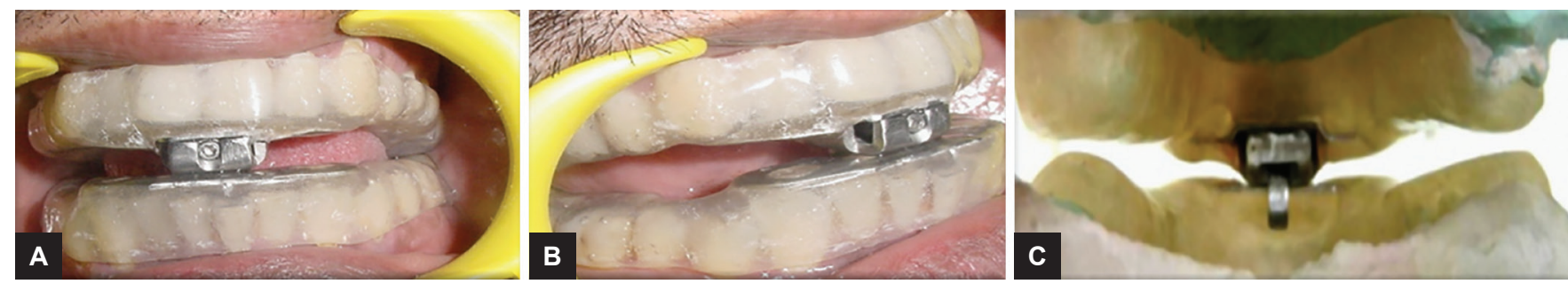

Figs 3A to C: Third-generation adjustable MAD (medical dental snoring appliance)
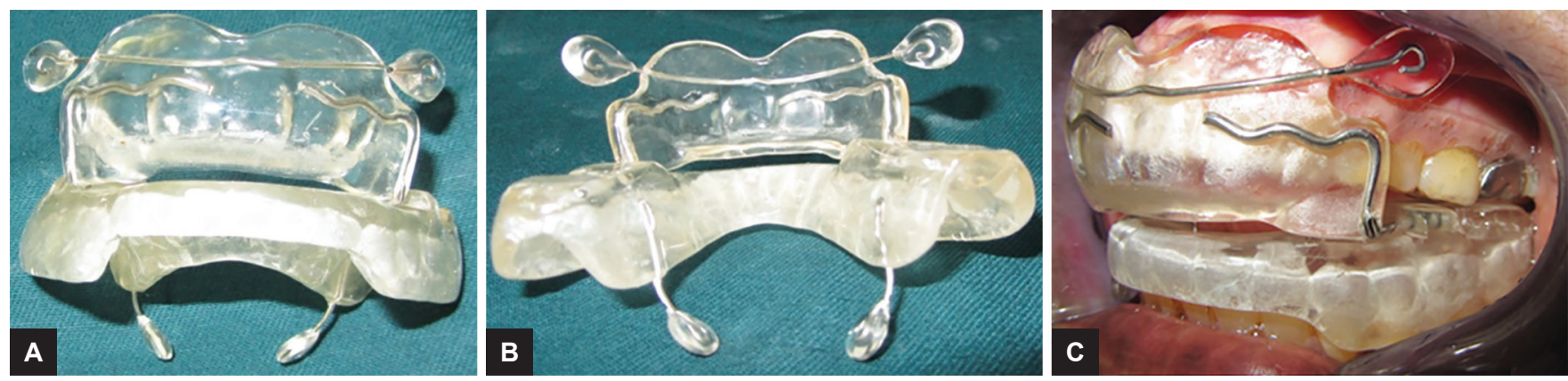

Figs 4A to C: Mandibular advancement splint with tongue lifters and nasal dilators

of Sleep Medicine guidelines for the management of SDB with OSA. ${ }^{30}$ Recently, the Ministry of Health and Family Welfare, Government of India, has published consensus report on management of OSA, named as Indian Initiative on OSA guidelines, which is a comprehensive document on guidelines management of OSA with OAT. ${ }^{31}$ A total of six severe cases of mandibular hypoplasia with severe OSA have been successfully managed with bilateral corpus mandibular distraction in the last 4 years. Two research papers connected with sleep medicine have been successfully completed and published. ${ }^{27,32}$ Three new projects are being undertaken, which includes appreciation of airway improvement with acoustic pharyngometry and rhinometry. These are the initial years and in the volume of cases are only going to increase. The efforts have been satisfying and requisite competence and capacity have been achieved. Our model may be considered for implementation in dental schools for inclusive growth and capacity building in this challenging discipline of health sciences.

\section{CONCLUSION}

The orthodontist now has an ever-increasing role in the recognition of patients who may be at risk for sleep disorders due to the fact that craniofacial factors like maxillomandibular deficiency, high arch palate, increased hyoid distance, and long face problems have been associated with upper airway collapsibility during sleep and the evaluation of the same is routine to them. Orthodontists can prevent, intercept, and correct SDB in select group of children and adults. There is a tremendous opportunity to interact with specialists in the field of sleep medicine and provision of oral appliances when indicated. In spite of the enormous epidemiological data determining the coexistence of SDB and impact on the health of a patient, orthodontic practitioners and trainers have not been able to integrate them in practice and postgraduate training. This communication is structured to provide an overview of the scope of dental sleep medicine and strategies to integrate the same in postgraduate training and practice to build capacity in this important discipline of health sciences. 


\section{REFERENCES}

1. Udwadia ZF, Doshi AV, Lonkar SG, Singh CI. Prevalence of sleep disordered breathing and sleep apnoea in middle aged urban Indian men. Am J Respir Crit Care Med 2004 Jan;169(2):168-173.

2. Lieberman P, Crelin E. On the speech of Neanderthal. Linguist Inq 1971;11(2):203-232.

3. Lavigne, GJ.; Morin, CM.; Carra, CM. The nature of sleep. In Lavigne GJ, Cistulli PA, Smith MT, editors. Sleep medicine for dentists-a practical overview. Hanover Park (IL): Quintessence Publishing; 2009. pp. 3-9.

4. Attanasio, R.; Bailey, DR. Medical and dental conditions related to sleep-related breathing disorders. In: Attanasio R, Bailey DR, editors. Dental management of sleep disorders. 1st ed. Ames (IA): Wiley Blackwell publishing; 2010. pp. 91-110.

5. Lopez-Jimenez F, Sert Kuniyoshi FH, Gami A, Somers VK. Obstructive sleep apnea: implications for cardiac and vascular disease. Chest 2008 Mar;133(3):793-804.

6. Somers VK, White DP, Amin R, Abraham WT, Costa F, Culebras A, Daniels S, Floras JS, Hunt CE, Olson LJ, et al. Sleep apnea and cardiovascular disease: an American Heart Association/American college of scientific statement from the American heart association council for high blood pressure research professional education committee, Council on clinical cardiology, stroke council and council for cardiovascular nursing. J Am Coll Cardiol 2008 Aug;52(8):686-717.

7. Pingitore G, Chrobak V, Petrie J. The social and psychological factors of bruxism. J Prosthet Dent 1991 Mar;65(3):443-446.

8. National Center on Sleep Disorders Research. Your guide to healthy sleep. Bethesda (MD): National Heart Lung and Blood Institutes of Health; 2005. p. 2.

9. Ohayon MM, Li KK, Guilleminault C. Risk factors for sleep bruxism in general population. Chest 2001 Jan;119(1):53-61.

10. Watanabe T, Isono S, Tanaka A, Tanzawa H, Nishino T. Contribution of body habitus and craniofacial characteristics to segmental closing pressure of the passive pharynx in patients with sleep disordered breathing. Am J Respir Crit Care Med 2002 Jan;165(2):260-265.

11. Hoehema A, Hovinga B, Stegenga B, De Bont LG. Craniofacial morphology and obstructive sleep apnea: a cephalometric analysis. J Oral Rehabil 2003 Jul;30(7):690-696.

12. Jayan B, Prasad BNBM, Atul K, Kharbanda OP, Roy Chowdhury SK, Gupta SH. The role of cephalometric analysis in obese and non obese urban Indian adults with obstructive sleep apnea syndrome: a pilot study. Indian J Sleep Med 2007 Mar;2(2):59-63.

13. Hang WA. Obstructive sleep apnea: dentistry's unique role in longevity enhancement. J Am Orthod Assoc 2007 Spring;7(2):28-32.

14. American Sleep Disorder Association Report. Practice parameters for the treatments of snoring and obstructive sleep apnea with oral devices. Sleep 1995 Jul;18(6):511-513.

15. Lowe, AA. Dental appliances for the treatments of snoring and obstructive sleep apnea. In: Kryger M, Roth T, Dement W, editors. Principles and practice of sleep medicine. 3rd ed. Philadelphia (PA): Saunders; 2000. pp. 929-939.

16. Lim J, Lasserson TJ, Fleetnam J, Wrignt J. Oral appliance for obstructive sleep apnea. Cochrane Database Syst Rev 2006 Jan;1:CD004435.
17. Ferguson KA, Ono T, Lowe AA, Keenan SP, Fleetham JA. A randomized cross over study of an oral appliance $v$ s nasal continuous positive airway pressure in treatment of mild moderate sleep apnea. Chest 1996 May;109(5):269-275.

18. Li KK, Riley RW, Powell NB, Guilleminault C. Maxillomandibular advancement for persistent obstructive sleep apnea after phase I surgery in patients without maxillomandibular deficiency. Laryngoscope 2000 Oct;110(10 Pt 1):1684-1688.

19. Ivanhoe JR, Attanasio R. Sleep disorders and oral devices. Dental Clin North Am 2001 Oct;45(4):735-758.

20. Learn Dental Sleep Medicine. www.sleepgroupsolutions.com. Accessed on June 5, 2017.

21. Menon S, Roy Chowdhury SK, Vasant MR. Distraction osteogenesis in the management of obstructive sleep apnea. Indian J Oral Maxillofac Surg 2003;18(3):25-29.

22. Jayan B, Prasad BN, Bhattacharyya D, Rajput AK, Dhiman RK. Management of case of severe obstructive sleep apnea with acrylic Herbst splint appliance: a case report. Indian J Sleep Med 2006;1(3):157-160.

23. Jayan B, Prasad BN, Bhattacharyya D, Rajput AK, Dhiman RK, Thampi PS. Management of obstructive sleep apnea with oral appliances: our experience. Indian J Sleep Med 2006 Jan;1(4):204-207.

24. Roy Chowdhury SK, Jayan B, Menon PS, Prasad BN, Ravishankar K. Management of obstructive sleep apnea and non apneic snoring with maxilla mandibular distraction osteogenesis. Indian J Sleep Med 2007;2(3):101-108.

25. Jayan B, Prasad BN, Kamat UR, Kharbanda OP, Bhattacharyya D. Therapeutic efficacy of Thorton adjustable positioner in management of patients with severe obstructive sleep apnea: a pilot study. Indian J Sleep Med 2008;3(3):97-101.

26. Jayan B, Prasad BN, Dhiman RK. Role of oral appliances in management of sleep disorders. Med J Armed Forces India 2009 Apr;65(2):123-127.

27. Nainan O, Jayan B, Chopra SS, Barthwal MS, Mukherjee M. Predictive morphometric model value estimation and its correlation with severity of sleep apnea in mixed Indian population: a pilot study. Indian J Sleep Med 2012 Jan;7(2):48-54.

28. Guilleminault C, Quo S, Huynh NT, Li K. Orthodontic expansion treatment and adenotonsillectomy in the treatment of obstructive sleep apnea in prepubertal children. Sleep 2008 Jul;31(7):953-957.

29. Pirelli P, Saponara M, De Rosa C, Fanucci E. Orthodontics and obstructive sleep apnea in children. Med Clin North Am 2010 May;94(3):517-529.

30. Ramar K, Dort LC, Katz SG, Lettieri CJ, Harrod CG, Thomas SM, Chervin RD. Clinical practice guideline for the treatment of obstructive sleep apnea and snoring with oral appliance therapy: an update for 2015. J Dent Sleep Med 2015 Jul;11(7):773-827.

31. Sharma SK, Katoch VM, Mohan A, Kadhiravan T, Elavarasi A, Ragesh R, Nischal N, Sethi P, Behera D, Bhatia M, et al. for Indian Initiative on Obstructive Sleep Apnoea (INOSA) Guidelines Working Group. Consensus \& evidence-based INOSA guidelines 2014 (first edition). Indian J Med Res 2014 Sep;140(3):451-468.

32. Sahoo NK, Jayan B, Chopra SS, Ramakrishnan S, Kochar G. Evaluation of upper airway dimensional changes and hyoid position following mandibular advancement in skeletal Class II cases: a cephalometric study. J Craniofac Surg 2012 Nov;23(6):36-41. 